\title{
Suppression of instabilities in multiphase flow by geometric confinement
}

\section{Citation}

Humphry, Katherine J., Armand Ajdari, Alberto Fernández-Nieves, Howard A. Stone, and David A. Weitz. 2009. "Suppression of Instabilities in Multiphase Flow by Geometric Confinement."

Physical Review E79 (5): 056310. https://doi.org/10.1103/PhysRevE.79.056310.

\section{Permanent link}

http://nrs.harvard.edu/urn-3:HUL.InstRepos:41511256

\section{Terms of Use}

This article was downloaded from Harvard University's DASH repository, and is made available under the terms and conditions applicable to Other Posted Material, as set forth at http:// nrs.harvard.edu/urn-3:HUL.InstRepos:dash.current.terms-of-use\#LAA

\section{Share Your Story}

The Harvard community has made this article openly available.

Please share how this access benefits you. Submit a story.

Accessibility 


\title{
Suppression of instabilities in multiphase flow by geometric confinement
}

\author{
Katherine J. Humphry, ${ }^{1}$ Armand Ajdari, ${ }^{2, *}$ Alberto Fernández-Nieves, ${ }^{1,3, \dagger}$ Howard A. Stone, ${ }^{3}$ and David A. Weitz ${ }^{1,3, \$}$ \\ ${ }^{1}$ Department of Physics, Harvard University, Cambridge, Massachusetts 02138, USA \\ 2 “Gulliver,” CNRS-ESPCI, UMR 7083, 75005 Paris, France \\ ${ }^{3}$ School of Engineering and Applied Sciences, Harvard University, Cambridge, Massachusetts 02138, USA
}

(Received 8 December 2008; published 18 May 2009)

\begin{abstract}
We investigate the effect of confinement on drop formation in microfluidic devices. The presence or absence of drop formation is studied for two immiscible coflowing liquids in a microfluidic channel, where the channel width is considerably larger than the channel height. We show that stability of the inner fluid thread depends on the channel geometry: when the width of the inner fluid is comparable to or larger than the channel height, hydrodynamic instabilities are suppressed, and a stable jet that does not break into drops results; otherwise, the inner fluid breaks into drops, in either a dripping or jetting regime. We present a model that accounts for the data and experimentally exploit this effect of geometric confinement to induce the breakup of a jet at a spatially defined location.
\end{abstract}

DOI: 10.1103/PhysRevE.79.056310

PACS number(s): 47.15.Fe, 47.20.Ma, 47.55.db

It has been demonstrated, both theoretically and experimentally, that otherwise quiescent cylinders of fluid are unstable and break into drops due to the surface-tension-driven Rayleigh-Plateau instability [1-3]. Conversely, their twodimensional analogs, ribbons of fluid, are stable and do not form drops [4]. Not surprisingly, the instability threshold is related to whether or not a perturbation increases or decreases the total interfacial area or energy. The transition between stable flow and drop-generating unstable flow, especially in channels with noncircular cross sections, is of particular interest for technologies that depend on controlled drop formation and manipulation, including many microfluidic applications [5-12]. For example, rapid manipulation and control of monodisperse drops $[13,14]$ is important for high-throughput screening of chemical and biological processes [15-17]. It has already been demonstrated that confining geometries affect the propagation of surface-tensiondriven instabilities. For example, the transition between twoand three-dimensional behavior in sheared polymer blends has been investigated $[18,19]$, as have the qualitative effects of channel cross section on jet stability in two-phase flows at low Reynolds numbers [20,21]. The stability of pressuredriven flow of two fluids in a circular pipe has been studied and was found to be unstable when the inner fluid was less viscous than the outer fluid [22]. However, the effects of a confining rectangular geometry on coflowing systems, such as those found in drop-making microfluidic devices, has not been extensively studied. Understanding the effects of confined channel geometry on fluid stability should provide further insight into the drop-making process in microfluidic systems and should lead to enhanced control of drop generation and manipulation.

In this paper we examine the effect of channel geometry in coflowing systems comprised of two immiscible liquids,

\footnotetext{
*armand.adjari@saint-gobain.com

'Present address: School of Physics, Georgia Institute of Technology, Atlanta, GA 30332, USA.

¥weitz@seas.harvard.edu
}

such as those commonly found in drop-making microfluidic devices, with an emphasis on the width and height of the channel. For these high aspect ratio systems, we find that when the width of the inner fluid is comparable to or larger than the height of the microfluidic channel, instabilities are suppressed and jet breakup does not occur while for smaller widths the device produces monodisperse drops. We account for this transition using a theoretical model of the two-phase flow and use changes in the confinement of a multiphase flow to trigger drop formation at a spatially defined position. We accomplish this control by changes in the channel height and width. The stability regime we document here is consistent with a geometric suppression of instabilities $[18,20]$.

We use standard soft lithographic techniques [23] to fabricate poly(dimethylsiloxane) (PDMS) microfluidic devices with channel widths much larger than channel heights using channel designs similar to those shown Fig. 1(a). All four walls of our device channels are made from identically prepared PDMS to ensure that wetting properties of all channel walls are the same. The important geometric dimensions of our devices are the height $h$, which is kept within the interval

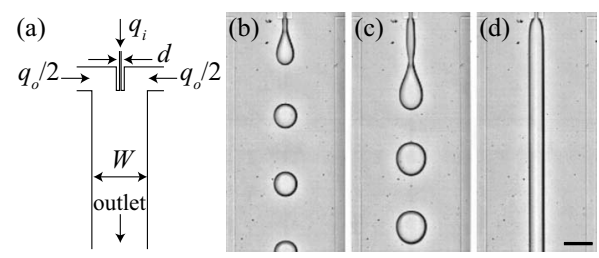

FIG. 1. (a) Schematic of a typical microfluidic device used in our experiments. The inner fluid enters through a nozzle of width $d$. The inner and outer fluids have volumetric flow rates $q_{i}$ and $q_{o}$, respectively. The devices have an outlet channel of width $W$. (b)-(d) High-speed camera images of the three observed behaviors. The microfluidic device shown in these images has dimensions $d=20 \mu \mathrm{m}, W=700 \mu \mathrm{m}$, and $h=34 \mu \mathrm{m}$. Liquids shown in these images have the properties $\eta_{o}=19.1 \mathrm{cP}, \quad \eta_{i}=3.4 \mathrm{cP}$, and $q_{o}=1600 \mu \mathrm{L} / \mathrm{hr}$. Scale bar=200 $\mu \mathrm{m}$. (b) Drop formation in a dripping regime $\left(q_{i}=160 \mu \mathrm{L} / \mathrm{hr}\right)$, (c) drop formation in a jetting regime $\left(q_{i}=320 \mu \mathrm{L} / \mathrm{hr}\right)$, and (d) jetting without breaking $\left(q_{i}=640 \mu \mathrm{L} / \mathrm{hr}\right)$. 
$[34,102] \mu \mathrm{m}$, and the width $W$, which is kept within the interval $[100,800] \mu \mathrm{m}$. In all cases dimensions are selected to ensure $W \gg h$; this results in quasi-two-dimensional outlet channels. It is this high aspect ratio cross-sectional geometry that has the effect of confining the fluid flow. The width $d$ of the nozzle for the inner fluid is within the interval $[20,160]$ $\mu \mathrm{m}$. We find that changes in $d$ have no effect on the experimental results reported here.

There are three fluid inputs to our devices: two side inlets for the outer fluid, a silicone oil (Dow Corning Corporation) with a viscosity $\eta_{o}=19.1$ or $95.9 \mathrm{cP}$, and a central inlet for the inner fluid, a fluorocarbon oil (3M) with viscosity $\eta_{i}$ $=3.6$ or $27.4 \mathrm{cP}$. These liquids allow us to access nearly 2 orders of magnitude of viscosity ratio: $0.038<\eta_{i} / \eta_{o}<1.4$. The silicone oil completely wets the channels; there is no wetting of the PDMS surface by the fluorocarbon oil. Surface tension $\gamma$ between the fluorocarbon and silicone oils was measured using the pendant drop method [24] and was found to fall in the range $[5.8,7.0] \times 10^{-3} \mathrm{~N} / \mathrm{m}$.

We perform our experiments by pumping the inner and outer liquids into the devices with flow rates of $q_{i}$ and $q_{o}$, respectively, using glass syringes (Hamilton Gastight) driven by constant flow-rate syringe pumps (Harvard Apparatus PHD 2000). In our experiments the Reynolds number of the inner fluid $\left(q_{i} / h \eta_{i}\right)$ ranges from $O(0.01-10)$ with flow rates of the fluids ranging from 1 to $10000 \mu \mathrm{L} / \mathrm{hr}$. The behavior of the fluid flow in the channel is recorded using a highspeed camera (Phantom, Vision Research Incorporated) mounted on an inverted microscope (Leica). Three distinct flow behaviors are observed: drop formation in a dripping regime [Fig. 1(b)], drop formation in a jetting regime [Fig. 1(c)], and jetting without breakup [Fig. 1(d)]. The first two behaviors, dripping and jetting with breakup, have been observed in other coflowing systems $[6,8,25,26]$ and are defined here as "unstable," as in both cases the drop formation is a result of hydrodynamic instabilities. The third behavior, jetting without breakup is only observed in a confined system and is defined here as "stable." We emphasize that in this regime the jet does not break, irrespective of channel length. In our experiments the channel length is typically $2 \mathrm{~cm}$ and always much larger than $W$.

We determine the stability of the inner fluid thread as we vary $q_{i}$ and $q_{o}$ over several orders of magnitude while holding the geometry and $\eta_{i} / \eta_{o}$ fixed using $\eta_{i} / \eta_{o} \simeq 0.038$ and $W / h \simeq 21$. We observe a transition from unstable (solid points) to stable (open points) behavior by holding $q_{o}$ constant and increasing $q_{i}$ or by holding $q_{i}$ constant and decreasing $q_{o}$, as shown in Fig. 2(a). We find that the boundary separating these two regimes occurs approximately at a constant $q_{i} / q_{o} \simeq 0.58$, corresponding to the straight line shown in Fig. 2(a).

To understand these results, we consider a stream of fluid of width $w_{i}$ flanked on either side by a second immiscible fluid in a channel of rectangular cross section, as in the case of the stable nonbreaking jet [Fig. 1(d)]. We assume fully developed laminar pressure-driven flow, which is strictly correct if the Reynolds numbers of both liquids are low enough and the flows are considered a distance greater than $h$ away from the nozzle. In the case that $h / W \leq 1$ it is reasonable to use Darcy's law to approximate the average fluid velocities of the two phases, $\bar{v}_{i}$ and $\bar{v}_{o}$ :
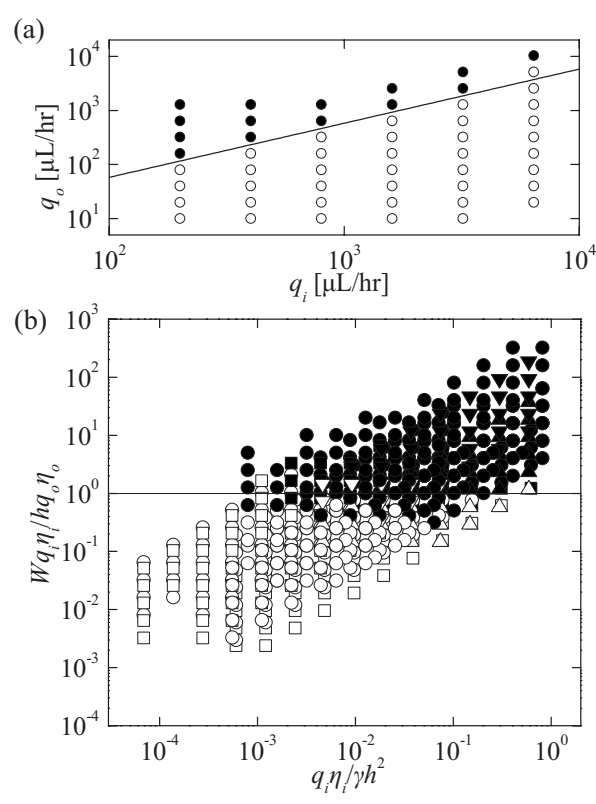

FIG. 2. Observed flow stability (a) as $q_{i}$ and $q_{o}$ are varied for $\eta_{i} / \eta_{o}=0.038$ in a channel with dimensions $h=34 \mu \mathrm{m}$ and $W=700 \mu \mathrm{m}$ and (b) for $\left(W q_{i} \eta_{i}\right) /\left(h q_{o} \eta_{o}\right)$ as a function of the inner fluid capillary number $\left(q_{i} \eta_{i}\right) /\left(\gamma h^{2}\right)$. The data include different channel heights, $h=34,102 \mu \mathrm{m}$, different channel widths, $W=100,200,300,400,700,800 \mu \mathrm{m}$, and different nozzle dimensions, $d=20,160 \mu \mathrm{m}$. Open symbols $(\bigcirc)$ indicate unstable flow with formation of drops [Figs. 1(b) and 1(c)]; filled symbols (-) indicate stable nonbreaking jets [Fig. 1(d)]. Symbol shapes correspond to viscosity ratios: $\square \eta_{i} / \eta_{o}=0.038 ; \bigcirc \eta_{i} / \eta_{o}=0.19 ; \triangle$ $\eta_{i} / \eta_{o}=0.29 ; \nabla n_{i} / n_{o}=1.4$.

$$
\begin{gathered}
\bar{v}_{i}=\kappa \frac{h^{2}}{\eta_{i}}\left|\frac{\partial p}{\partial x}\right|=\frac{q_{i}}{h w_{i}}, \\
\bar{v}_{o}=\kappa \frac{h^{2}}{\eta_{o}}\left|\frac{\partial p}{\partial x}\right|=\frac{q_{o}}{h\left(W-w_{i}\right)},
\end{gathered}
$$

where $\kappa$ is a constant prefactor that depends on the geometry of the device and $\partial p / \partial x$ is the pressure gradient in the channel along the direction of flow. It is assumed that the pressure is constant in directions perpendicular to the flow. Solving Eqs. (1) and (2) for the width of the inner stream, $w_{i}$, gives

$$
w_{i} \approx \frac{\frac{q_{i} \eta_{i}}{q_{o} \eta_{o}}}{1+\frac{q_{i} \eta_{i}}{q_{o} \eta_{o}}} W .
$$

We hypothesize that the stability of the inner fluid is determined by the geometric confinement of the flow and that surface-tension-driven breakup occurs when the width of the inner fluid, $w_{i}$, is comparable to or smaller than the channel height $h$. In this case, the inner fluid can adopt a circular cross section and hydrodynamic instabilities are present. When $w_{i}$ is greater than $h$ the inner fluid cannot form a cylindrical shape and breakup does not occur; instabilities are suppressed. This hypothesis is consistent with theory and 
observations that demonstrate that unconfined threedimensional cylinders of fluid break into drops due to the Rayleigh-Plateau instability [1,2], while ribbons of fluid, the two-dimensional analog of cylinders of fluid, are stable as all perturbations to their shape increase their surface area $[4,19]$.

Our hypothesis suggests that stable nonbreaking jets are expected when $w_{i}>h$. Applying this result to Eq. (3) in the limit of $W / h \gg 1$ suggests that stable jets should result when

$$
\frac{\frac{q_{i} \eta_{i}}{q_{o} \eta_{o}}}{1+\frac{q_{i} \eta_{i}}{q_{o} \eta_{o}}} \frac{W}{h} \simeq \frac{q_{i} \eta_{i}}{q_{o} \eta_{o}} \frac{W}{h}>1 .
$$

The transition from unstable to stable flow occurs for a constant value of $q_{i} / q_{o}$ when all other variables are held constant, establishing a linear relationship between $q_{i}$ and $q_{o}$, which is qualitatively in agreement with experimental observations [Fig. 2(a)]. Using the experimental parameters for the data showed in Fig. $2(\mathrm{a}), \eta_{i} / \eta_{o} \simeq 0.038$ and $W / h \simeq 21$, we find that the inner fluid should form a stable jet when $q_{i} / q_{o}>1.25$, which is within a factor of about 2 of the experimentally determined value of 0.58 .

To further confirm our theoretical stability criterion, we perform experiments for a wide range of flow-rate ratios, viscosity ratios, and channel aspect ratios. We plot the results in Fig. 2(b) in terms of the variable $\left(W q_{i} \eta_{i}\right) /\left(h q_{o} \eta_{o}\right)$ against the capillary number of the inner fluid. The capillary number for the inner fluid is known to be important for the hydrodynamic stability for jets formed in low-Reynoldsnumber two-phase flows [21]. We observe that all of the data sets scale together, which confirms that when $\left(W q_{i} \eta_{i}\right) /\left(h q_{o} \eta_{o}\right)>1$ the flow is a stable unbroken jet, while when $\left(W q_{i} \eta_{i}\right) /\left(h q_{o} \eta_{o}\right)<1$ drops are formed. The transition from stable to unstable flow does not occur at exactly $\left(W q_{i} \eta_{i}\right) /\left(h q_{o} \eta_{o}\right)=1$ for all data sets, presumably because our method for calculating $w_{i}$ is somewhat crude. These results support our hypothesis that geometric confinement suppresses hydrodynamic instabilities under flow conditions by forcing the inner fluid to assume a noncircular cross section and, consequently, that the transition from unstable to stable behavior occurs when $w_{i} \simeq h$. Furthermore, we show that the stability of the flow can be controlled by varying $q_{i} / q_{o}$ for different viscosity ratios.

Our model also predicts the width of the jet in the stable regime [Eq. (3)]. We compare this prediction (dashed line) with experimental measurements of $w_{i}$ (solid symbols) in Fig. 3. As expected, the width of the jet increases with increasing $q_{i}$ and $\eta_{i}$ and decreases with increasing $q_{o}$ and $\eta_{o}$. There is good agreement between experiment and theory for the larger values of $q_{i} \eta_{i} / q_{o} \eta_{o}$ that we explore. However, Eq. (3) fails to predict the lower values of $w_{i}$ we measure. In this regime $w_{i}$ is comparable to $h$, and the parallel-flow calculations in Eqs. (1)-(3) are too simplistic, as the flow velocities at the interface between the two fluids and at the walls become important. It is then necessary to correct Eq. (3) to include interfacial and wall effects.

To include interface and wall effects in an exact calculation requires a numerical solution because the detailed shape

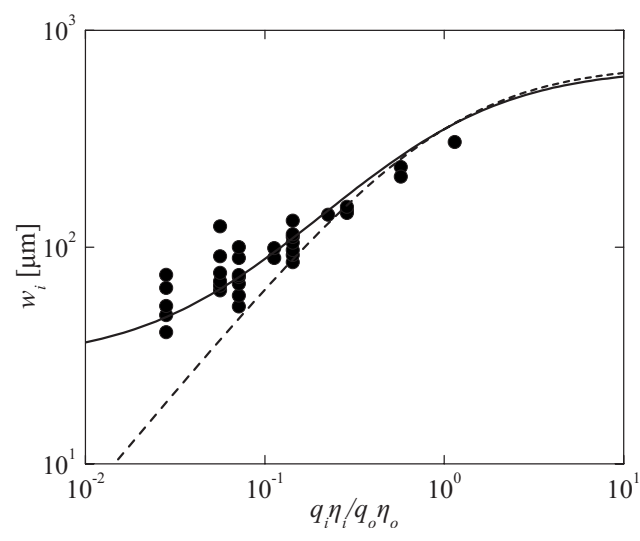

FIG. 3. Measured values of the width of the inner fluid, $w_{i}$, compared with predictions from Eq. (3) (dashed line) and Eq. (5) (solid line) for $h=34 \mu \mathrm{m}$ and $W=700 \mu \mathrm{m}$.

is unknown, so instead we identify the dominant geometric corrections by considering a region of size $h \times h$ on either side of the interface and beside the walls. Balancing the shear stresses at the interface gives the interfacial velocity $v_{I}=\left(\eta_{o} \bar{v}_{o}+\eta_{i} \bar{v}_{i}\right) /\left(\eta_{o}+\eta_{i}\right)$ [27]. The inner and outer flow rates are then approximately $q_{i}=\bar{v}_{i} w_{i} h+\left(v_{I}-\bar{v}_{i}\right) h^{2}$ and $q_{o}$ $=\bar{v}_{o}\left(W-w_{i}\right) h+\left(v_{I}-2 \bar{v}_{o}\right) h^{2}$, respectively. We further assume that $\eta_{i} / \eta_{o} \ll 1$, as is the case in most of our experiments, and use Darcy's law to obtain

$$
w_{i} \approx \frac{W \frac{q_{i} \eta_{i}}{q_{o} \eta_{o}}+h\left(1-\frac{q_{i} \eta_{i}}{q_{o} \eta_{o}}\right)}{1+\frac{q_{i} \eta_{i}}{q_{o} \eta_{o}}} .
$$

This more complete expression for $w_{i}$ correctly describes the width of the nonbreaking jet throughout the experimental range, as shown by the solid line in Fig. 3. As $w_{i} / h \rightarrow 1$, the experimentally measured values of $w_{i}$, as well as those predicted by Eq. (5), are significantly larger than those predicted by Eq. (3). This provides some understanding for the experimentally observed stable jets below $\left(W q_{i} \eta_{i}\right) /\left(h q_{o} \eta_{o}\right)=1$ in Fig. 2(a). Alternatively, a downward bowing of the roof of the PDMS channel, lowering the effective value of $h$, would also reduce the value of $\left(W q_{i} \eta_{i}\right) /\left(h q_{o} \eta_{o}\right)$ at which the transition from unstable to stable flow occurs. However, the transition from unstable to stable flow is robust over several orders of magnitude of flow rate, indicating that this is not the underlying cause.

The understanding gained from experiments and theory can be exploited to induce breakup of a stable jet by changing the aspect ratio of the channel and thus the confinement of the inner fluid. Based on the parameters that control the transition between the unstable and stable regimes, we fabricated a microfluidic device where the height of the outlet channel abruptly increases. The first section of the channel has height $h_{1}=36 \mu \mathrm{m}$, while the second section of the channel has height $h_{2}=86 \mu \mathrm{m}$. The selection of heights guarantees that there exists a range of $\left(q_{i} \eta_{i}\right) /\left(q_{o} \eta_{o}\right)$ such that $\left(W q_{i} \eta_{i}\right) /\left(h_{2} q_{o} \eta_{o}\right)<1<\left(W q_{i} \eta_{i}\right) /\left(h_{1} q_{o} \eta_{o}\right)$, which predicts a stable jet in the first section of the outlet channel and an 


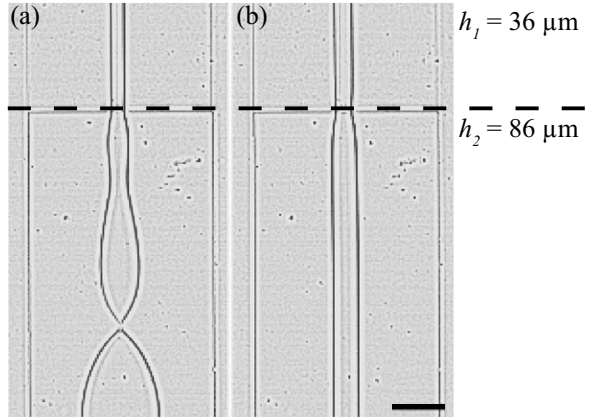

FIG. 4. High-speed images of fluid flow in a channel with an abrupt increase in outlet channel height. $\eta_{i}=3.6 \mathrm{cP}, \eta_{o}=19.1 \mathrm{cP}$, $W=700 \mu \mathrm{m}$, and $q_{o}=800 \mu \mathrm{L} / \mathrm{hr}$. A dashed line indicates the position of the height change. Scale bar $=200 \mu \mathrm{m}$. (a) Drop formation at step $\left(q_{i}=160 \mu \mathrm{L} / \mathrm{hr}\right)$ and (b) stable jetting for the entire channel length $\left(q_{i}=320 \mu \mathrm{L} / \mathrm{hr}\right)$.

unstable flow and drop formation in the second section of the outlet channel. The flow behavior should transition from stable to unstable at the point where the channel height abruptly increases.

The results of these experiments confirm our prediction of an instability of the jet triggered by a step increase in the channel height as shown in Fig. 4(a). This result demonstrates that when the confinement of the inner fluid is decreased, in this case by increasing the channel height, a transition from stable to unstable fluid flow occurs. If, in the same device, we further increase $q_{i}$, so that $w_{i}>h_{2}$, we recover the stable regime along the entire length of the outlet channel, as shown in Fig. 4(b). These experiments provide insight into a mechanism for controlling drop breakup at a specific position in the channel $[12,28]$.

Equation (4) suggests that it should also be possible to transition from stable to unstable flow by decreasing the channel width $W$. We fabricated a microfluidic device where the width of the outlet channel abruptly decreases. The first section of the channel has width $W_{1}=200 \mu \mathrm{m}$, while the second section of the channel has width $W_{2}=100 \mu \mathrm{m}$. The results of our experiments demonstrate, as expected, that there exists three stability regimes: drop breakup

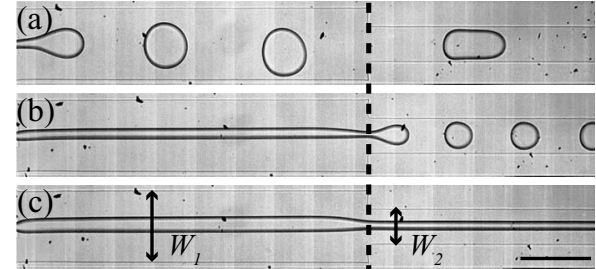

FIG. 5. High-speed images of fluid flow in a channel with an abrupt decrease in outlet channel width. $\eta_{i}=3.6 \mathrm{cP}, \eta_{o}=19.1 \mathrm{cP}$, $W_{1}=200 \mu \mathrm{m}, W_{2}=100 \mu \mathrm{m}, h=25 \mu \mathrm{m}$, and $q_{o}=100 \mu \mathrm{L} / \mathrm{hr}$. A dashed line indicates the position of the width change. Scale bar $=200 \mu \mathrm{m}$. (a) Drop formation at inlet $\left(q_{i}=20 \mu \mathrm{L} / \mathrm{hr}\right)$, (b) drop formation at width decrease $\left(q_{i}=40 \mu \mathrm{L} / \mathrm{hr}\right)$, and (c) stable jetting for the entire channel length $\left(q_{i}=80 \mu \mathrm{L} / \mathrm{hr}\right)$.

at the inlet, for the case when $\left(W_{1} q_{i} \eta_{i}\right) /\left(h q_{o} \eta_{o}\right)<1$ [Fig. 5(a)]; stable flow in the first section of the channel that becomes unstable and breaks into drops when the width of the channel abruptly changes, for the case when $\left(W_{2} q_{i} \eta_{i}\right) /\left(h q_{o} \eta_{o}\right)<1<\left(W_{1} q_{i} \eta_{i}\right) /\left(h q_{o} \eta_{o}\right) \quad[$ Fig. 5(b) $]$; and flow that is stable along the entire length of the channel, for the case when $\left(W_{2} q_{i} \eta_{i}\right) /\left(h q_{o} \eta_{o}\right)>1$ [Fig. 5(c)]. Thus, we have demonstrated that it is possible to transition from a stable to an unstable flow by increasing the channel height (Fig. 4) or by decreasing the channel width (Fig. 5).

In conclusion, we have shown that hydrodynamic instabilities of the inner fluid of a two-phase flow can be suppressed using geometric confinement and that changes in the geometric confinement along the length of a multiphase flow can result in drop formation at a specified position. Our study further suggests additional routes for geometric control of interfaces and instabilities in multiphase flows [14]. There clearly remain opportunities for more studies of this type and for quantification of the different instabilities we observe.

This work was supported by the NSF (Grant No. DMR0602684) and by the Harvard MRSEC (Grant No. DMR0820484). We thank Michael Brenner, Andrew Utada, and Srinivas Parachuri for helpful discussions. A.F.-N. acknowledges University of Almería and support from DPI 200806624-C03-03.
[1] Lord Rayleigh, Proc. R. Soc. London 29, 71 (1879).

[2] Lord Rayleigh, Proc. London Math. Soc. S1-10, 4 (1878).

[3] J. Eggers, Rev. Mod. Phys. 69, 865 (1997).

[4] M. San Miguel, M. Grant, and J. D. Gunton, Phys. Rev. A 31, 1001 (1985).

[5] H. A. Stone, A. D. Stroock, and A. Ajdari, Annu. Rev. Fluid Mech. 36, 381 (2004).

[6] G. F. Christopher and S. L. Anna, J. Phys. D 40, R319 (2007).

[7] T. Thorsen, R. W. Roberts, F. H. Arnold, and S. R. Quake, Phys. Rev. Lett. 86, 4163 (2001).

[8] S. L. Anna, N. Bontoux, and H. A. Stone, Appl. Phys. Lett. 82, 364 (2003).

[9] M. He, J. S. Edgar, G. D. M. Jeffries, R. M. Lorenz, J. P.
Shelby, and D. T. Chiu, Anal. Chem. 77, 1539 (2005).

[10] J. E. Reiner, A. M. Crawford, R. B. Kishore, L. S. Goldner, K. Helmerson, and M. K. Gilson, Appl. Phys. Lett. 89, 013904 (2006).

[11] Y.-C. Tan, K. Hettiarachchi, M. Siu, Y.-R. Pan, and A. Lee, J. Am. Chem. Soc. 128, 5656 (2006).

[12] C. Priest, S. Herminghaus, and R. Seemann, Appl. Phys. Lett. 88, 024106 (2006).

[13] K. Ahn, C. Kerbage, T. P. Hunt, R. M. Westervelt, D. R. Link, and D. A. Weitz, Appl. Phys. Lett. 88, 024104 (2006).

[14] D. R. Link, S. L. Anna, D. A. Weitz, and H. A. Stone, Phys. Rev. Lett. 92, 054503 (2004).

[15] J.-U. Shim, G. Cristobal, D. R. Link, T. Thorsen, Y. Jia, K. 
Piattelli, and S. Fraden, J. Am. Chem. Soc. 129, 8825 (2007).

[16] B. Zheng, J. D. Tice, and R. F. Ismagilov, Anal. Chem. 76, 4977 (2004).

[17] A. Sgro, P. Allen, and D. Chiu, Anal. Chem. 79, 4845 (2007).

[18] K. B. Migler, Phys. Rev. Lett. 86, 1023 (2001).

[19] Y. Son, N. Martys, J. Hagedorn, and K. Migler, Macromolecules 36, 5825 (2003).

[20] P. Guillot, A. Colin, and A. Ajdari, Phys. Rev. E 78, 016307 (2008).

[21] P. Guillot, A. Colin, A. S. Utada, and A. Ajdari, Phys. Rev. Lett. 99, 104502 (2007).

[22] C. E. Hickox, Phys. Fluids 14, 251 (1971).
[23] D. C. Duffy, J. C. McDonald, O. A. Schueller, and G. M. Whitesides, Anal. Chem. 70, 4974 (1998).

[24] O. I. del Río and A. W. Neumann, J. Colloid Interface Sci. 196, 136 (1997).

[25] C. Cramer, P. Fischer, and E. J. Windhab, Chem. Eng. Sci. 59, 3045 (2004).

[26] A. S. Utada, A. Fernandez-Nieves, H. A. Stone, and D. A. Weitz, Phys. Rev. Lett. 99, 094502 (2007).

[27] A. Nadim, A. Borhan, and H. Haj-Hariri, J. Colloid Interface Sci. 181, 159 (1996).

[28] S. Sugiura, M. Nakajima, and M. Seki, Langmuir 18, 5708 (2002). 